\title{
THE BENDING OF A WORK-HARDENING CIRCULAR PLATE BY A UNIFORM TRANSVERSE LOAD*
}

\author{
BY \\ WILLIAM E. BOYCE \\ Brown University
}

\begin{abstract}
This paper contains an analysis of the bending moments and deflection of a work-hardening circular plate under the action of a uniformly distributed transverse load. A segment-wise linear yield condition and the associated flow rules are used in order to avoid the unsound features of total stress-strain laws while retaining much of their mathematical simplicity.

1. Introduction. For several years investigators in the field of work-hardening plastic solids have been aware of the physical shortcomings of total stress-strain laws (see, for example, [1] ${ }^{* *}$ ). Consequently all recent theoretical work has involved incremental laws. However, since total laws are mathematically more tractable than incremental laws, solutions to many specific problems are still based on the total laws. In such papers a plausibility argument is given to indicate that the results are approximately correct, despite their questionable derivation.

W. Prager [2] has recently suggested a compromise which provides, to a large extent at least, the mathematical advantages of total stress-strain laws, while retaining the fundamental character of incremental laws. This is accomplished through the use of a segmentwise linear yield condition together with the associated flow rules. In order to make this paper self-contained a brief summary of this method is given in Sec. 2 .

In Sec. 3 the bending of a uniformly loaded, simply supported circular plate is studied. For uniaxial stress the mechanical behavior of the rigid work-hardening plate material
\end{abstract}

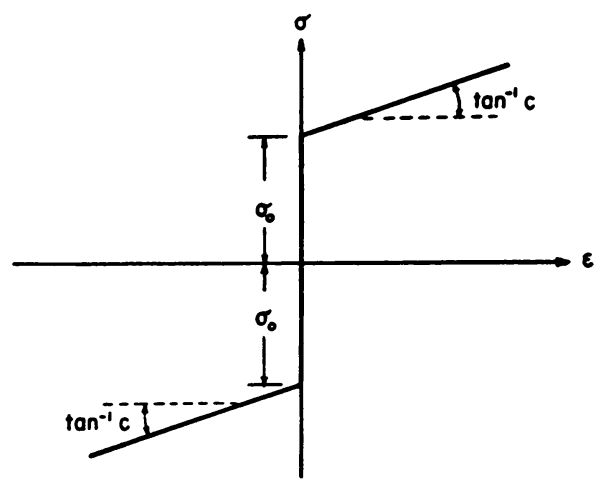

FIG. 1.

is indicated in Fig. 1. For plane stress with the principal stresses $\sigma_{1}$ and $\sigma_{2}$, the yield condition is assumed to be given by the polygon in Fig. 3 .

2. Review. Figure 2 depicts a simple model which represents the mechanical behavior of work-hardening plastic solids in plane stress with fixed principal axes. Let $\sigma_{1}, \sigma_{2}$,

${ }^{*}$ Received Oct. 24, 1955.

**Numbers in square brackets refer to the Bibliography at the end of the paper. 
$\epsilon_{1}, \epsilon_{2}$ denote the principal stresses and strains respectively. The elliptical frame $F$ is mounted so that it can undergo any translation in its plane. The perfectly smooth pin $P$ is free to move inside the frame and may impart motion to the frame only when in contact with it. If there is no friction between frame and pin, such motion will always have the direction of the exterior normal to the frame at the point of contact. As the frame is displaced by the moving pin, the vector $0 P$ has the components $\sigma_{1}$ and $\sigma_{2}$, while the vector $O C$ has the components $c \epsilon_{1}$ and $c \epsilon_{2}, c$ being the slope of the stressstrain diagram in Fig. 1. The elliptical frame corresponds to the yield condition of $v$. Mises, which in plane stress takes the form

$$
\sigma_{1}^{2}+\sigma_{2}^{2}-\sigma_{1} \sigma_{2}=\sigma_{0}^{2},
$$

where $\sigma_{0}$ is the yield stress in simple tension.

A model of the sort just described clearly represents an incremental stress-strain law in which the state of strain at any time depends not only on the state of stress at that time but also on the loading history. It is precisely at this point that incremental laws may become extremely cumbersome, as the loading process must be followed step by step in order to determine the final states of stress and strain.

The mechanical behavior indicated by the model of Fig. 2 will not change sub-

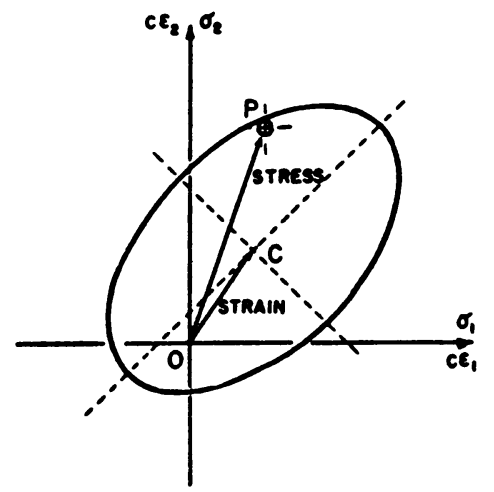

Fia. 2.

stantially if the elliptical frame is replaced by a polygonal frame, as in Fig. 3, provided that the latter has a sufficient number of sides. Either inscribed or circumscribed polygonal approximations may be employed.

There is, however, a significant mathematical difference between the nature of the flows corresponding to the two types of frames. As long as the pin pushes against a given side of the polygonal frame, the translation of the frame is in the direction of the exterior normal. Consequently, the instantaneous position of the frame depends only on: (1) the time at which the pin made contact with the side in question, and (2) the instantaneous position of the pin. Similarly, as long as the pin engages a given corner of the frame, the displacement of the frame coincides with that of the pin. Hence the instantaneous position of the frame depends only on: (1) the time at which the pin first engaged the given corner, and (2) the instantaneous position of the pin.

In this way a segmentwise linear yield condition and the associated flow rules result in the partial independence of the tinal strain from the loading history. This permits the use of total stress-strain laws "in the small". Since the pin will usually contact 
several sides and corners of the frame during the loading process, several types of total laws may be required in succession. Thus the fundamental nature of incremental laws is retained "in the large":

3. Bending of work-hardening plates. Consider a circular plate of radius $R$ and uniform thickness $h$ made of a rigid, work-hardening material. Let the plate be simply supported around the entire edge, and suppose that it is subjected to a uniform transverse load of intensity $p$ per unit area. The usual approximations of the technical theory of the bending of plates allow the state of stress and strain at any point to be specified by the radial and circumferential bending moments, $M$ and $N$, and the radial and circumferential curvatures, $\kappa$ and $\lambda$. The bending moments must satisfy the equation of equilibrium

$$
(r M)^{\prime}-N=-\frac{1}{2} p r^{2},
$$

where the prime denotes differentiation with respect to $r$. In terms of the deflection $w$ of the middle surface of the plate, the curvatures are given by

$$
\kappa=-w^{\prime \prime}, \quad \lambda=-\frac{1}{r} w^{\prime} .
$$

On the basis of these definitions $\kappa$ and $\lambda$ must satisfy a compatibility equation, namely,

$$
(r \lambda)^{\prime}=\kappa .
$$

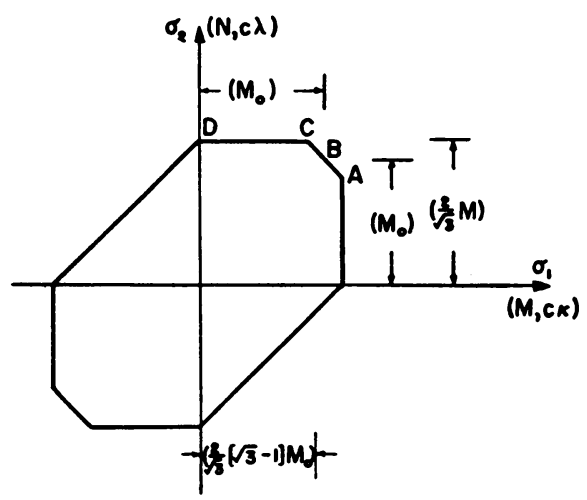

FIG. 3.

The relation between bending moments and curvatures is indicated by the pinframe model in Fig. 3, where the labels in parentheses must be used. It will be useful to speak of plastic states represented by the interior of the side $C D$, for example, as regime $C D$, and similarly for the other sides and vertices of the yield polygon.

The problem now falls naturally into two parts: first, the determination of the critical load $p_{0}$, the bending moment distribution, and the velocity field at the onset of plastic flow; second, the determination of the bending moments and deflection at some later time, that is, for $p>p_{0}$.

At the beginning of plastic flow no work-hardening has taken place, and the analysis may be carried out by well-known methods of the theory of rigid, perfectly plastic solids $[3,4]$.

3.1. Incipient plastic flow. At the onset of plastic flow, $M=N=M_{0}$ at the center of the plate, so that the center is in regime $B$ (see Fig. 3). In the neighborhood of the 
center it is easy to show from the equilibrium equation that regime $B C$ applies. At some radius $r=\rho$, regime $C$ is reached, and for $r>\rho$ regime $C D$ occurs. Since $M(R)=0$ the edge of the plate is in regime $D$. The yield condition and flow rules in these regimes are:

$$
\begin{aligned}
& r \leq \rho: \frac{2}{3^{1 / 2}}\left(3^{1 / 2}-1\right) M_{0} \leq M \leq M_{0}, \quad N=2 M_{0}-M, \quad d_{\kappa}=d \lambda, \\
& r \geq \rho: 0 \leq M \leq \frac{2}{3^{1 / 2}}\left(3^{1 / 2}-1\right) M_{0}, \quad N=\frac{2}{3^{1 / 2}} M_{0}, \quad d_{\kappa}=0,
\end{aligned}
$$

where $d \lambda$ and $d \kappa$ are the increments of curvature corresponding to an increment $d p$ of the load.

The yield conditions (5) and (6) can be substituted into the equilibrium equation to give two differential equations for $M$, one valid in $0 \leq r \leq \rho$, the other in $\rho \leq r \leq R$. Their integration is subject to the boundary conditions $M(0)=M_{0}, M(R)=0$, so that

$$
M= \begin{cases}M_{0}-\frac{1}{8} p_{0} r^{2}, & r \leq \rho \\ \frac{2}{3^{1 / 2}} M_{0}\left(1-\frac{R}{r}\right)+\frac{1}{6} p_{0} R^{2}\left(\frac{R}{r}-\frac{r^{2}}{R^{2}}\right), & r \geq \rho .\end{cases}
$$

The transition point $\rho$ and critical load $p_{0}$ are obtained from the fact that

$$
M(\rho)=\frac{2}{3^{1 / 2}}\left(3^{1 / 2}-1\right) M_{0},
$$

because at $r=\rho$ the plate must be in regime $C$. Combination of (9) with (7) and (8) furnishes the equations

$$
\begin{gathered}
\frac{\rho^{2}}{R^{2}}=8\left(\frac{2}{3^{1 / 2}}-1\right) \frac{M_{0}}{p_{0} R^{2}}, \\
\frac{\rho^{3}}{R^{3}}-12\left(\frac{2}{3^{1 / 2}}-1\right) \frac{M_{0}}{p_{0} R^{2}} \frac{\rho}{R}+\frac{12}{3^{1 / 2}} \frac{M_{n}}{p_{0} R^{2}}-1=0
\end{gathered}
$$

in the unknowns $\rho / R$ and $M_{0} /\left(p_{0} R^{2}\right)$. The solutions are found to be

$$
\begin{aligned}
& \frac{\rho}{R}=0.431, \\
& p_{0}=6.66 \frac{M_{0}}{R^{2}} .
\end{aligned}
$$

It now remains to be shown that there exists a velocity field for the incipient plastic flow that is compatible with the stress field just obtained. This is necessary to insure that the preceding statical analysis provides the actual value of $p_{0}$ and not merely a lower bound. If the dot superscript denotes differentiation with respect to time (which here may be identified with differentiation with respect to the monotonically increasing load intensity $p$ ), such a velocity field must satisfy the following relations, the first 
two of which spring from the flow rules (5), (6):

$$
\left.\begin{array}{rr}
w^{\prime \prime}-\frac{1}{r} w^{\circ \prime}=0, & r \leq \rho, \\
w^{\prime \prime \prime}=0 & r \geq \rho, \\
w^{\cdot}=0, & r=R,
\end{array}\right\}
$$

As is readily verified, the following velocity field satisfies these conditions:

$$
w^{\cdot}= \begin{cases}w_{0}^{\cdot}\left[1-\frac{r}{2 \rho R-\rho^{2}}\right], & r \leq \rho, \\ w_{0}^{\cdot} \frac{R-r}{R-\rho / 2}, & r \geq \rho .\end{cases}
$$

3.2. Work-hardening effects. For a work-hardening material, plastic flow will continue only under increasing loads. For $p>p_{0}$, the region of transition between regimes $B C$ and $C D$ enlarges from a circle into an annulus which is in regime $C$. The situation is as indicated in Fig. 4. Zones 1 and 4 contain material which has been in regimes $B C$

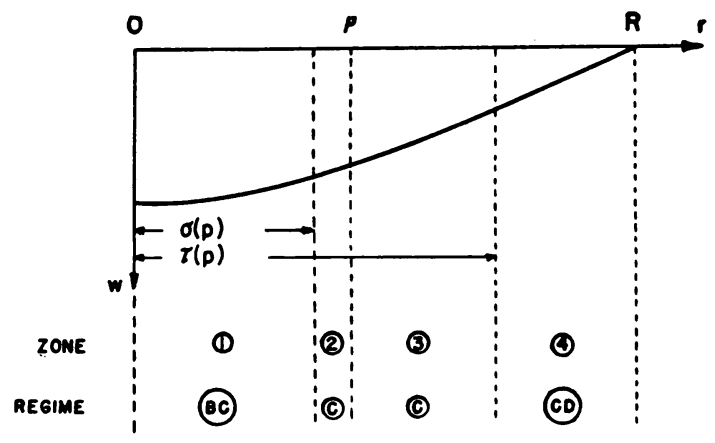

FIG. 4.

and $C D$ respectively since the beginning of plastic flow; on the other hand, the material in zones 2 and 3 is now in regime $C$ but was originally in regimes $B C$ and $C D$, respectively. Each regime must be considered separately.

Zones 2 and 3. Elements of the plate in zones 2 and 3 are now in regime $C$ but at the onset of plastic flow were in either regime $B C$ or regime $C D$. Under many loading programs the relation between the total curvatures and bending moments for such an element is the same as if the element had always been in regime $C$, provided that the initial conditions at the appropriate point of $B C$ or $C D$ are replaced by those at $C$. Permissible types of loading are those for which the pin always remains engaged in a given corner of the frame, once it has become so engaged. After a solution has been obtained on this basis, it is necessary to verify that this restriction is indeed satisfied. Figure 5 will help to clarify this point.

Since in regime $C$ the pin is engaged in the corner $C$ of the frame, the motions of pin and frame are identical. Hence

$$
d M=c d \kappa, \quad d N=c d \lambda .
$$


In view of the above observation, (16) may be integrated subject to the initial conditions

$$
M=\frac{2}{3^{1 / 2}}\left(3^{1 / 2}-1\right) M_{0}, \quad N=\frac{2}{3^{1 / \overline{2}}} M_{0},
$$

giving

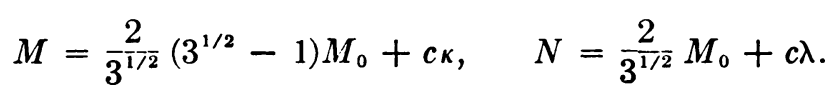

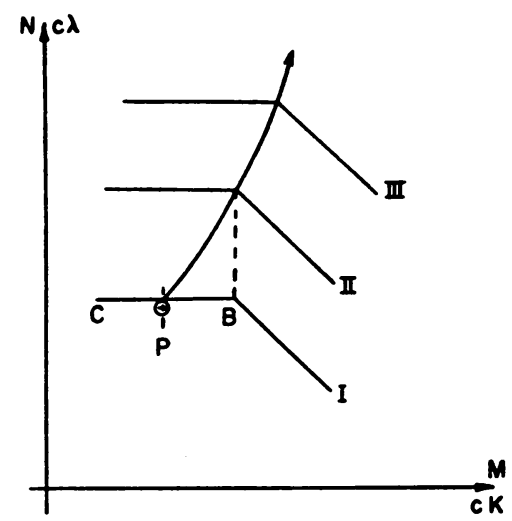

Frg. 5.

I. Initial position of frame.

II. Position of frame when pin $\mathrm{P}$ engages corner $\mathrm{B}$.

III. Later position of frame.

Eliminating $\kappa$ and $\lambda$ among (18) and the compatibility equation (4) furnishes

$$
r N^{\prime}=M-N+\frac{2}{3^{1 / 2}}\left(2-3^{1 / 2}\right) M_{0},
$$

which must be solved simultaneously with the equilibrium equation (2).

Boundary conditions are obtained from consideration of the transition points between regime $C$ and regimes $B C$ and $C D$. The boundary between regimes $B C$ and $C$ (zones 1 and 2) will occur, say at $r=\sigma(p)$, when the pin reaches the straight line through $C$ normal to $B C$; that is, when

$$
M-N=-\frac{2}{3^{1 / 2}}\left(2-3^{1 / 2}\right) M_{0}, \quad r=\sigma .
$$

Similarly, the boundary between regimes $C$ and $C D$ (zones 3 and 4) is reached, at a point $r=\tau(p)$, when the pin touches the straight line through $C$ normal to $C D$; that is, when

$$
M=\frac{2}{3^{1 / 2}}\left(3^{1 / 2}-1\right) M_{0}, \quad r=\tau .
$$

Now subtracting (19) from (2) and integrating with respect to (20) gives

$$
M-N=-\frac{1}{8} p r^{2}-\frac{1}{3^{1 / 2}}\left(2-3^{1 / 2}\right) M_{0}+\frac{\sigma^{2}}{r^{2}}\left[\frac{1}{8} p \sigma^{2}-\frac{1}{\left.3^{1 / 2}\left(2-3^{1 / 2}\right) M_{0}\right] .}\right.
$$


Substituting (22) into (2) and integrating subject to (21) furnishes

$$
\begin{aligned}
M=\frac{2}{3^{1 / 2}}\left[3^{1 / 2}-1\right] M_{0} & +\frac{1}{2\left(3^{1 / 2}\right)}\left(2-3^{1 / 2}\right) M_{0}\left[2 \ln \frac{r}{\tau}-\frac{\sigma^{2}}{r^{2}}+\frac{\sigma^{2}}{\tau^{2}}\right] \\
& +\frac{1}{16} p\left[3 \tau^{2}-3 r^{2}+\frac{\sigma^{4}}{r^{2}}-\frac{\sigma^{4}}{\tau^{2}}\right],
\end{aligned}
$$

and hence

$$
N=M_{0}+\frac{1}{2\left(3^{1 / 2}\right)}\left(2-3^{1 / 2}\right) M_{0}\left[2 \ln \frac{r}{\tau}+\frac{\sigma^{2}}{r^{2}}+\frac{\sigma^{2}}{\tau^{2}}\right]+\frac{p}{16}\left[3 \tau^{2}-r^{2}-\frac{\sigma^{4}}{r^{2}}-\frac{\sigma^{4}}{\tau^{2}}\right] .
$$

Equations (23) and (24) can now be used to show that the condition on the type of loading mentioned earlier in this section is, in fact, satisfied.

Zone 1. Elements in zone 1 have been in regime $B C$ since the onset of plastic flow. The flow rule (5) can thus be integrated to give $\kappa=\lambda$, and then the compatibility equation becomes $r \lambda^{\prime}=0$. Hence

$$
\kappa=\lambda=f(p) .
$$

A further relation valid for regime $B C$ is obtained from the fact that, as long as the pin pushes against the side $B C$ of the frame, the displacement of the frame equals the component of the pin displacement that is normal to $B C$. This kinematical relation is expressed by

$$
c \kappa \Delta M+c \lambda \Delta N=c^{2}\left(\kappa^{2}+\lambda^{2}\right)
$$

or, by writing out $\Delta M$ and $\Delta N$ in full and using (25), by

$$
M(p)-M\left(p_{0}\right)+N(p)-N\left(p_{0}\right)=2 c f(p) .
$$

Now at the beginning of flow, (5) states that

$$
M\left(p_{0}\right)+N\left(p_{0}\right)=2 M_{0} ;
$$

therefore (26) becomes

$$
M+N-2 M_{0}=2 c f(p) .
$$

The function $f(p)$ is obtained by using the values of $M$ and $N$ at $r=\sigma$ as furnished by (23) and (24). Thus

$$
\begin{aligned}
2 c f(p)=\frac{1}{3^{1 / 2}}\left(2-3^{1 / 2}\right) M_{0}\left(2 \ln \frac{\sigma}{\tau}+\frac{\sigma^{2}}{\tau^{2}}\right)-\frac{1}{3^{1 / 2}}\left(2-3^{1 / 2}\right) M_{0} & \\
& +\frac{1}{8} p\left(3 \tau^{2}-2 \sigma^{2}-\frac{\sigma^{4}}{\tau^{2}}\right),
\end{aligned}
$$

and

$$
\begin{aligned}
M+N=\left(3-\frac{2}{3^{1 / 2}}\right) M_{0}+\frac{1}{3^{1 / 2}}\left(2-3^{1 / 2}\right) M_{0}\left(2 \ln \frac{\sigma}{\tau}\right. & \left.+\frac{\sigma^{2}}{\tau^{2}}\right) \\
& +\frac{1}{8} p\left(3 \tau^{2}-2 \sigma^{2}-\frac{\sigma^{4}}{\tau^{2}}\right)
\end{aligned}
$$


Finally, substituting from (29) into the equilibrium equation, and integrating under the restriction that $M$ be finite at $r=0$ provides

$$
\begin{aligned}
M=\frac{1}{2}\left(3-\frac{2}{3^{1 / 2}}\right) M_{0}+\frac{1}{2\left(3^{1 / 2}\right)}\left(2-3^{1 / 2}\right) M_{0} & \left(2 \ln \frac{\sigma}{\tau}+\frac{\sigma^{2}}{\tau^{2}}\right) \\
& +\frac{1}{16} p\left(3 \tau^{2}-2 \sigma^{2}-\frac{\sigma^{4}}{\tau^{2}}\right)-\frac{1}{8} p r^{2} .
\end{aligned}
$$

Then

$$
\begin{aligned}
N=\frac{1}{2}\left(3-\frac{2}{3^{1 / 2}}\right) M_{0}+\frac{1}{2\left(3^{1 / 2}\right)}\left(2-3^{1 / 2}\right) M_{0} & \left(2 \ln \frac{\sigma}{\tau}+\frac{\sigma^{2}}{\tau^{2}}\right) \\
& +\frac{1}{16} p\left(3 \tau^{2}-2 \sigma^{2}-\frac{\sigma^{4}}{\tau^{2}}\right)+\frac{1}{8} p r^{2} .
\end{aligned}
$$

It is clear that the symmetry condition $M=N$ at $r=0$ is satisfied.

Furthermore, (20) supplies a relation between $\sigma, \tau$, and $p$, provided that values of $M$ and $N$ from (30) and (31) are used; this equation is

$$
\frac{\sigma^{2}}{R^{2}}=\frac{\rho^{2}}{R^{2}} \frac{p_{n}}{p}
$$

when (10) has also been employed.

Zone 4. The material in zone 4 has been in regime $C D$ since plastic flow began; hence the flow rule (6) may be integrated, giving $\kappa=0$. The compatibility equation then becomes

$$
r \lambda^{\prime}+\lambda=0
$$

so that

$$
\lambda=\frac{1}{r} g(p) .
$$

The kinematical relation valid in zone 4 is obtained from the fact that the displacement of the frame equals the vertical component of the pin displacement, that is,

$$
d N=c d \lambda \text {. }
$$

The initial value of the circumferential bending moment is given by (6) while the circumferential curvature vanishes when the pin first makes contact with the frame. Thus in zone 4

$$
N=\frac{2}{3^{1 / 2}} M_{0}+c \lambda=\frac{2}{3^{1 / 2}} M_{0}+\frac{c}{r} g(p) .
$$

The function $g(p)$ may be obtained from the value of $N$ at $r=\tau$ as given by (24). Hence

$$
\frac{c}{\tau} g(p)=\frac{1}{3^{1 / 2}}\left(2-3^{1 / 2}\right) M_{0} \frac{\sigma^{2}}{\tau^{2}}-\frac{1}{3^{1 / 2}}\left(2-3^{1 / 2}\right) M_{0}+\frac{1}{8} p\left(\tau^{2}-\frac{\sigma^{4}}{\tau^{2}}\right)
$$


and (34) then gives

$$
N=\frac{2}{3^{1 / 2}} M_{0}-\frac{1}{3^{1 / 2}}\left(2-3^{1 / 2}\right) \frac{M_{0}}{\tau r}\left(\tau^{2}-\sigma^{2}\right)+\frac{1}{8} \frac{p}{\tau r}\left(\tau^{4}-\sigma^{4}\right) .
$$

The equilibrium equation now provides an expression for $M$, in which the integration is carried out subject to $M=0$ at $r=R$, as required by the conditions of simple support. Thus

$$
\begin{aligned}
M=\frac{2}{3^{1 / 2}} M_{0}\left(1-\frac{R}{r}\right)-\frac{1}{3^{1 / 2}}\left(2-3^{1 / 2}\right) & \frac{M_{0}}{\tau r}\left(\tau^{2}-\sigma^{2}\right) \ln \frac{r}{R} \\
& +\frac{1}{8} \frac{p}{\tau r}\left(\tau^{4}-\sigma^{4}\right) \ln \frac{r}{R}+\frac{1}{6} \frac{p}{r}\left(R^{3}-r^{3}\right) .
\end{aligned}
$$

A second relation between $\sigma, \tau$, and $p$ is gotten from (21) by using the value of $M(\tau)$ from (37),

$$
\begin{aligned}
\frac{2}{3^{1 / 2}} \frac{M_{0}}{p R^{2}}\left(2-3^{1 / 2}-\frac{R}{\tau}\right) & +\frac{1}{8}\left(\frac{\tau^{2}}{R^{2}}-\frac{\sigma^{4}}{\tau^{2} R^{2}}\right) \ln \frac{\tau}{R} \\
& -\frac{1}{3^{1 / 2}}\left(2-3^{1 / 2}\right) \frac{M_{0}}{p R^{2}}\left(1-\frac{\sigma^{2}}{\tau^{2}}\right) \ln \frac{\tau}{R}+\frac{1}{6}\left(\frac{R}{\tau}-\frac{\tau^{2}}{R^{2}}\right)=0 .
\end{aligned}
$$

Now only the displacement of each element of the plate remains to be found. Using the definition of $\lambda$ in terms of the displacement $w$, differential equations for $w$, one valid in each regime, are furnished by (25), (18), and (33) as follows:

$$
\left.\begin{array}{rlrl}
0 & \leq r \leq \sigma: & w^{\prime} & =-r f(p) \\
\sigma & \leq r \leq \tau: & -\frac{c}{r} w^{\prime} & =N(p)-\frac{2}{3^{1 / 2}} M_{0} \\
\tau & \leq r \leq R: & w^{\prime} & =-g(p)
\end{array}\right\} .
$$

Of course, the functions $f(p), N(p)$, and $g(p)$ are those given by (28), (24), and (35) respectively. The integration of (39) must be carried out subject to the boundary condition $w=0$ at $r=R$ and the requirement that $w$ be continuous at $r=\sigma$ and $r=\tau$. The symmetry condition $w^{\prime}=0$ at $r=0$ is found to be automatically satisfied. The appropriate displacements are the following:

$$
\begin{aligned}
& 0 \leq r \leq \sigma: \\
& \frac{c w}{M_{0} R^{2}}=-\frac{1}{2\left(3^{1 / 2}\right)}\left(2-3^{1 / 2}\right)\left[\left(\frac{\sigma^{2}}{R^{2}}+\frac{r^{2}}{R^{2}}\right) \ln \frac{\sigma}{\tau}\right. \\
& \left.+\left(\frac{\tau^{2}}{R^{2}}-\frac{\sigma^{2}}{R^{2}}\right)\left(2 \frac{R}{\tau}-\frac{1}{2}\right)-\frac{1}{2} \frac{r^{2}}{R^{2}}\left(1-\frac{\sigma^{2}}{\tau^{2}}\right)\right] \\
& +\frac{1}{64} \frac{p_{0} R^{2}}{M_{0}} \frac{p}{p_{0}}\left[3 \frac{\sigma^{4}}{R^{4}}-3 \frac{\tau^{4}}{R^{4}}-8 \frac{\sigma^{4}}{\tau R^{3}}+8 \frac{\tau^{3}}{R^{3}}+4 \frac{\sigma^{4}}{R^{4}} \ln \frac{\sigma}{\tau}\right. \\
& \left.-4 \frac{r^{2}}{R^{2}}\left(\frac{3}{2} \frac{\tau^{2}}{R^{2}}-\frac{\sigma^{2}}{R^{2}}-\frac{1}{2} \frac{\sigma^{4}}{R^{2} \tau^{2}}\right)\right]
\end{aligned}
$$


$\sigma \leq r \leq \tau:$

$$
\begin{aligned}
\frac{c w}{M_{0} R^{2}}=-\frac{1}{2\left(3^{1 / 2}\right)}\left(2-3^{1 / 2}\right) & {\left[\left(\frac{r^{2}}{R^{2}}+\frac{\sigma^{2}}{R^{2}}\right) \ln \frac{r}{\tau}+\frac{3}{2}\left(\frac{\sigma^{2}}{R^{2}}-\frac{r^{2}}{R^{2}}\right)\right.} \\
& \left.+2 \frac{\tau}{R}-\frac{1}{2} \frac{\tau^{2}}{R^{2}}+\frac{1}{2} \frac{\sigma^{2} r^{2}}{\tau^{2} R^{2}}-2 \frac{\sigma^{2}}{\tau R}\right] \\
& +\frac{1}{64} \frac{p_{0} R^{2}}{M_{0}} \frac{p}{p_{0}}\left[4 \frac{\sigma^{4}}{R^{4}} \ln \frac{r}{\tau}+\frac{r^{4}}{R^{4}}+6 \frac{\sigma^{4}}{R^{4}}-3 \frac{\tau^{4}}{R^{4}}\right. \\
& \left.-6 \frac{r^{2} \tau^{2}}{R^{4}}+2 \frac{r^{2} \sigma^{4}}{\tau^{2} R^{4}}-8 \frac{\sigma^{4}}{\tau R^{3}}+8 \frac{\tau^{3}}{R^{3}}\right]
\end{aligned}
$$

$\tau \leq r \leq R$

$$
\frac{c w}{M_{0} R^{2}}=-\frac{1}{3^{1 / 2}}\left(2-3^{1 / 2}\right)\left(\frac{R}{\tau}-\frac{r}{\tau}\right)\left(\frac{\tau^{2}}{R^{2}}-\frac{\sigma^{2}}{R^{2}}\right)-\frac{1}{8} \frac{p_{0} R^{2}}{M_{0}} \frac{p}{p_{0}}\left(\frac{R}{\tau}-\frac{r}{\tau}\right)\left(\frac{\sigma^{4}}{R^{4}}-\frac{\tau^{4}}{R^{4}}\right) .
$$

4. Numerical calculations and conclusions. Since the yield condition used in this investigation is represented by a polygon circumscribed to the Mises ellipse, the corresponding critical load $p_{0}$ at which plastic flow begins is an upper bound for the actual critical load predicted by the Mises theory. The latter quantity has been found by Hopkins and Wang [4] to be equal to $6.52\left(M_{0} / R^{2}\right)$, whereas the analysis of Sec. 2 yields the present approximate value $6.66\left(M_{0} / R^{2}\right)$.

Equations (32) and (38) have been used to compute $\sigma / R$ and $\tau / R$ for $p / p_{0}=1.25$, 1.50. The results are shown in Table 1. Values of the normalized bending moments and deflection have also been computed for various values of $r / R$ and are given in Table 2 .

\begin{tabular}{|c|c|c|c|c|c|c|c|}
\hline & $r / R$ & 0 & 0.2 & 0.4 & 0.6 & 0.8 & 1.0 \\
\hline$p / p_{0}=1.00$ & $\begin{array}{l}M / M_{0} \\
N / M_{0} \\
c w / M_{0} R^{2}\end{array}$ & $\begin{array}{l}1 \\
1 \\
0\end{array}$ & $\begin{array}{l}0.96670 \\
1.0333 \\
0\end{array}$ & $\begin{array}{l}0.86679 \\
1.1332 \\
0\end{array}$ & $\begin{array}{l}0.68076 \\
1.15470 \\
0\end{array}$ & $\begin{array}{l}0.38850 \\
1.15470 \\
0\end{array}$ & $\begin{array}{l}0 \\
1.15470 \\
0\end{array}$ \\
\hline$p / p_{0}=1.25$ & $\begin{array}{l}M / M_{0} \\
N / M_{0} \\
c w / M_{0} R^{2}\end{array}$ & $\begin{array}{l}1.3179 \\
1.3179 \\
0.12223\end{array}$ & $\begin{array}{l}1.2762 \\
1.3595 \\
0.11587\end{array}$ & $\begin{array}{l}1.1511 \\
1.4724 \\
0.096802\end{array}$ & $\begin{array}{l}0.90163 \\
1.4310 \\
0.066685\end{array}$ & $\begin{array}{l}0.51128 \\
1.3631 \\
0.033348\end{array}$ & $\begin{array}{l}0 \\
1.3214 \\
0\end{array}$ \\
\hline$p / p_{0}=1.50$ & $\begin{array}{l}M / M_{0} \\
N / M_{0} \\
c w / M_{0} R^{2}\end{array}$ & $\begin{array}{l}1.6234 \\
1.6234 \\
0.24907\end{array}$ & $\begin{array}{l}1.5735 \\
1.6734 \\
0.23661\end{array}$ & $\begin{array}{l}1.4208 \\
1.7754 \\
0.19922\end{array}$ & $\begin{array}{l}1.1089 \\
1.7132 \\
0.13984\end{array}$ & $\begin{array}{l}0.62912 \\
1.5937 \\
0.070242\end{array}$ & $\begin{array}{l}0 \\
1.5059 \\
0\end{array}$ \\
\hline
\end{tabular}
These quantities are also portrayed by the solid curves in Figs. 6(a), (b), (c). The dotted

TABLE 1

\begin{tabular}{c||c|c|c}
\hline \hline$p / p_{0}$ & 1 & 1.25 & 1.5 \\
\hline$\sigma / R$ & 0.43103 & 0.38552 & 0.35194 \\
$\tau / R$ & 0.43103 & 0.63363 & 0.71801 \\
\hline
\end{tabular}

TABLE 2 


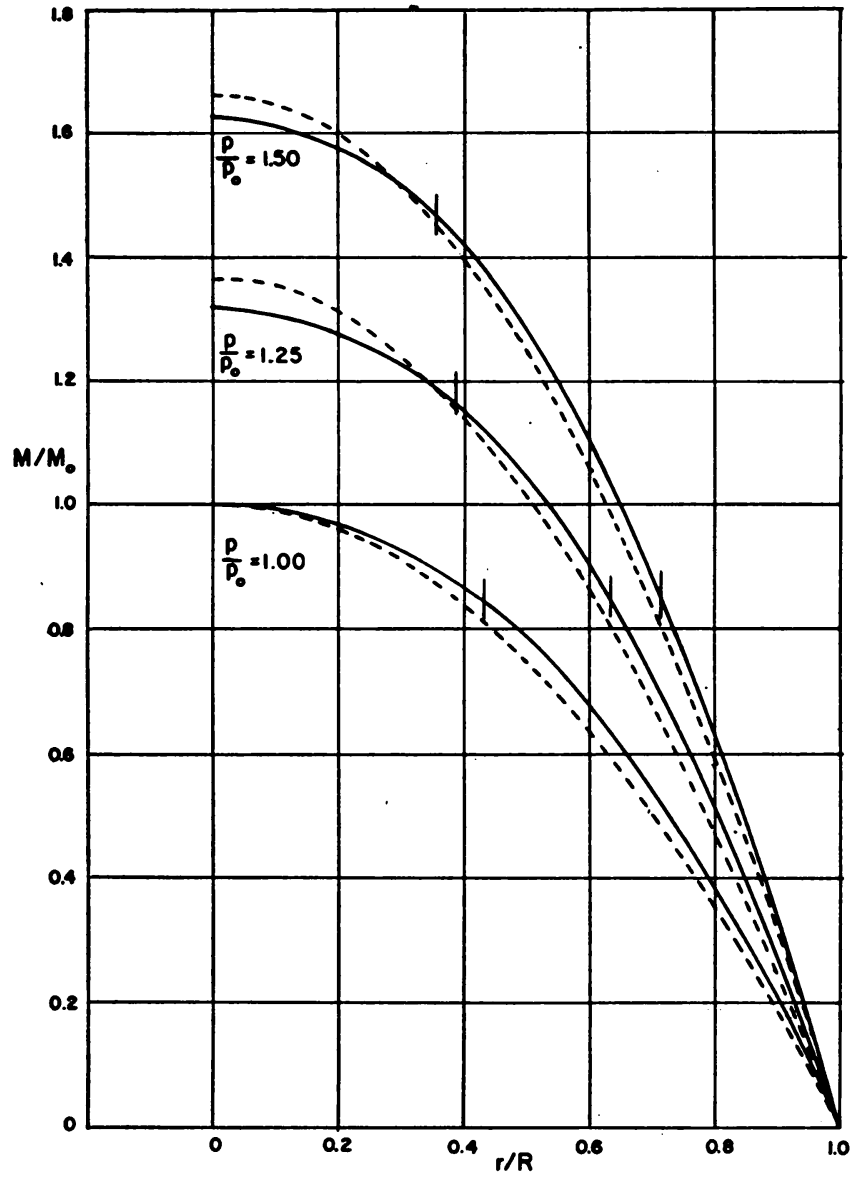

FIG. 6a.

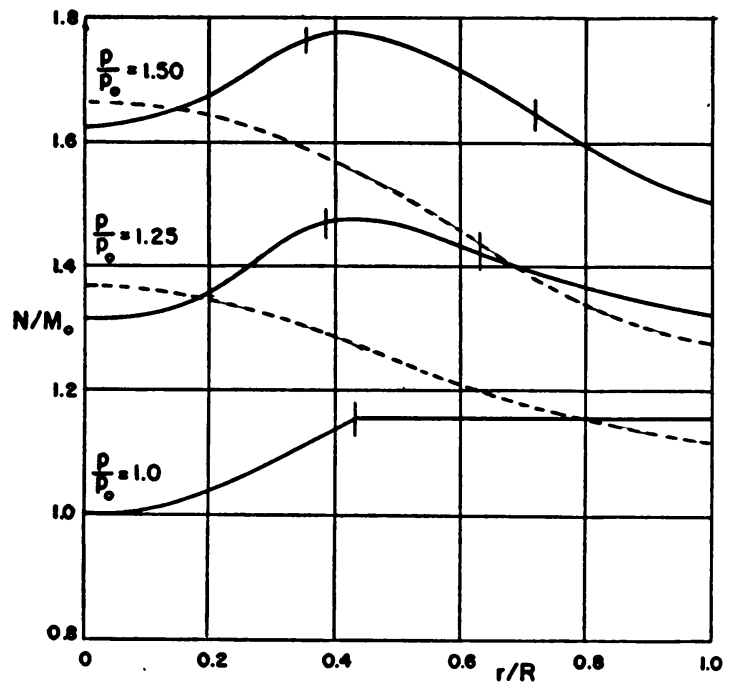

FIG. $6 \mathrm{~b}$. 


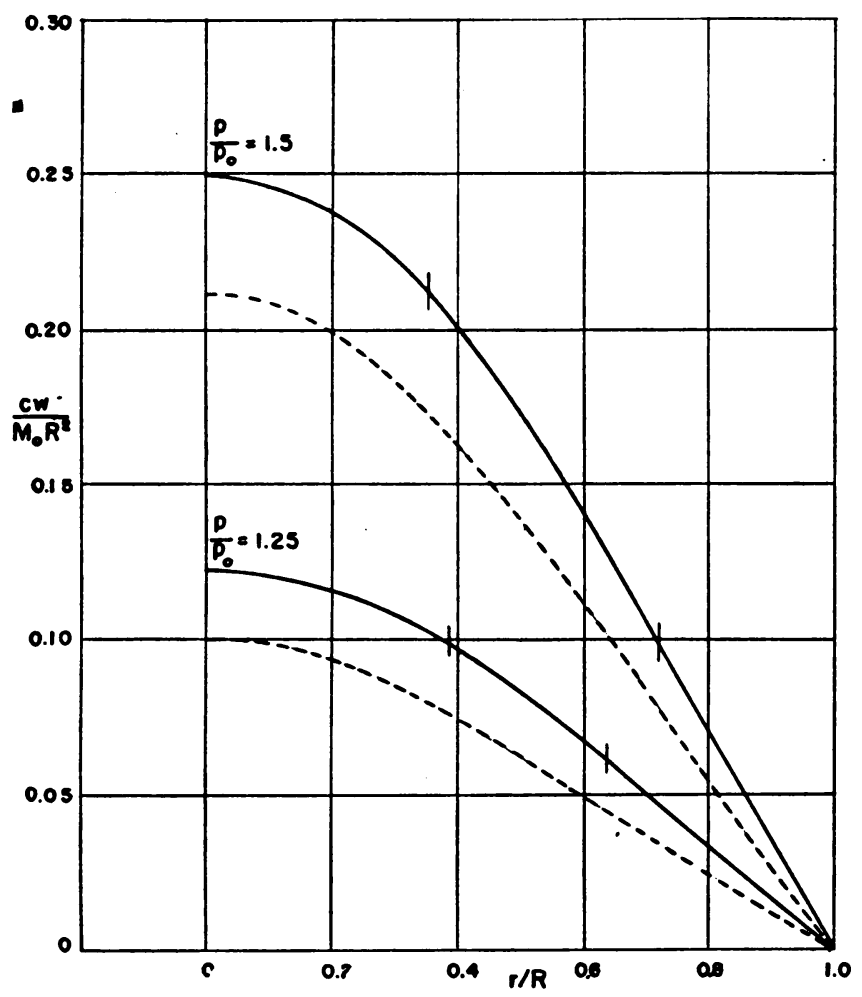

Fig. 6c.

curves are those obtained by Prager [2] with the Tresca hexagon inscribed to the Mises ellipse as the yield condition. The radial bending moment distribution is quite similar in the two cases, while the circumferential bending moment and displacement exhibit more substantial variations.

Acknowledgments. The results presented in this paper were obtained in the course of research sponsored by the Office of Naval Research under Contract Nonr-562(10) with Brown University. The author would like to express his sincere appreciation to Professor W. Prager for his helpful discussions during this investigation. He would also like to thank Mrs. R. C. Alverson for her aid with the computational aspects of the problem.

\section{BIBLIOGRAPHY}

1. W. Prager, Theory of plastic flow versus theory of plastic deformation, J. Appl. Phys. 19, 540-543 (1948)

2. W. Prager, A new method of analyzing stresses and strains in work-hardening plastic solids, Brown University Report A11-123, 1955

3. H. G. Hopkins and W. Prager, The load-carrying capacities of circular plates, J. Mech. Phys. Solids 2, $1-13(1953)$

4. H. G. Hopkins and A. G. Wang, Load-carrying capacities for circular plates of perfectly-plastic material with arbitrary yield condition, J. Mech. Phys. Solids 3, 117-129 (1954) 